\title{
IMPROVING CLASSIFICATION FOR BRAIN COMPUTER INTERFACES USING TRANSITIONS AND A MOVING WINDOW
}

\author{
Ricardo Aler, Inés M. Galván, José M. Valls \\ Computer Science Department, Universidad Carlos III de Madrid, Avda. Universidad, 30, Leganés, Spain \\ aler@inf.uc3m.es, igalvan@inf.uc3m.es, jvalls@inf.uc3m.es
}

Keywords: Brain Computer Interface, Machine learning.

\begin{abstract}
The context of this paper is the brain-computer interface (BCI), and in particular the classification of signals with machine learning methods. In this paper we intend to improve classification accuracy by taking advantage of a feature of BCIs: instances run in sequences belonging to the same class. In that case, the classification problem can be reformulated into two subproblems: detecting class transitions and determining the class for sequences of instances between transitions. We detect a transition when the Euclidean distance between the power spectra at two different times is larger than a threshold. To tackle the second problem, instances are classified by taking into account, not just the prediction for that instance, but a moving window of predictions for previous instances. Our results are competitive with those obtained in the BCI III competition.
\end{abstract}

\section{INTRODUCTION}

The context of this paper is the brain-computer interface (BCI), a growing research field, that would allow users to control computers and other devices by means of brain signals (Curran and Stokes, 2003), (Millan J del R and Gerstner, 2004) (G. Pfurtscheller and Birbaumer, 2005), (Kubler and Muller, 2007). One of the main problems of $\mathrm{BCI}$ is to accurately decode individual brain signals. Machine Learning techniques are typically applied here, by training classifiers with the brain signals of the user that is going to use the interface (Dornhege et al., 2007). For instance, a BCI can be trained to recognize three different classes corresponding to three different mental states: left-hand motor imagery, right-hand motor imagery, and object rotation imagery.

Noisy weak signals and high variability between same-user sessions ${ }^{1}$ make the classification problem difficult, resulting in many on-line classification errors, frustated users, and low transfer (speed) rates from the user to the computer. The transfer rate could be increased if three or more classes are used. How-

\footnotetext{
${ }^{1}$ This means that the classifier learned during one session might not be accurate in the next session, even for the same user.
}

ever, multi-class classification problems are more difficult than two-class ones.

In this paper we intend to improve classification accuracy by taking advantage of a feature of BCIs: instances run in sequences belonging to the same class (for instance, right-hand motor imagery), followed by a transition into a different class. Typically, the BCI classification problem is tackled by trying to classify the EEG signal at every instant in time. However, given that classes run in sequences, the classification problem can be reformulated into two subproblems:

- Detecting class transitions

- Determining the class for sequences of instances between transitions.

The first problem can be approached in many different ways. In this paper we detect transitions by computing the Euclidean distance between the power spectra at two different times (samples) and signalling a class transition when the distance is larger than a threshold.

By detecting transitions, the accuracy of the second problem (classification between transitions) can also be improved in two ways. First, if the class of the last sequence (before the transition) is known, that class can be discarded after the transition, hence be- 
coming a simpler N-1 class classification problem. Also, sequences may contain many instances (depending of the sampling rate), but only a few are necessary to determine the class of the whole sequence. This is actually no different than determining if a coin is biased towards heads or tails, based on a small sample of coin tosses. We use a similar idea in this paper. We have applied these ideas to the data supplied for the BCI III competition. The datasets are described in Section 2. The method is described in Section 3. Section 4 shows the obtained results. Section 5 summarizes our results and draws some conclusions.

\section{DESCRIPTION OF EEG DATA}

In this paper we are going to use a high quality dataset acquired in the IDIAP Research Institute by Silvia Chiappa and Jos del R. Milln (Millán, 2004). It was used in the BCI-III competition that took place in 2005 . $^{2}$ This dataset contains data from 3 normal subjects during 4 non-feedback sessions. The subjects sat in a normal chair, relaxed arms resting on their legs. There are 3 tasks, so this is a three-class classification problem:

- Imagination of repetitive self-paced left hand movements

- Imagination of repetitive self-paced right hand movements

- Generation of words beginning with the same random letter

All 4 sessions of a given subject were acquired on the same day, each lasting 4 minutes with 5-10 minutes breaks in between them. The subject performed a given task for about 15 seconds and then switched randomly to another task at the operator's request. EEG data is not splitted in trials since the subjects are continuously performing any of the mental tasks. Data was provided in two ways: raw EEG signals, and data with precomputed features. In this paper, we use the precomputed dataset.

Features were precomputed as follows. The raw EEG potentials were first spatially filtered by means of a surface Laplacian. Then, every 62.5 ms (i.e., 16 times per second) the power spectral density (PSD) in the band $8-30 \mathrm{~Hz}$ was estimated over the last second of data with a frequency resolution of $2 \mathrm{~Hz}$ for the 8 centro-parietal channels $\mathrm{C} 3, \mathrm{Cz}, \mathrm{C} 4, \mathrm{CP} 1, \mathrm{CP} 2$, $\mathrm{P} 3, \mathrm{Pz}$, and P4. As a result, an EEG sample is a 96dimensional vector (8 channels times 12 frequency components).

\footnotetext{
${ }^{2}$ http : //ida.first.fraunhofer.de/projects /bci/competition_iii/.
}

In summary, in this paper we are going to tackle a three-class classification problem with 96 input attributes, which define the PSD over $62.5 \mathrm{~ms}$ for 8 input channels. There are three subjects, with four datasets (sessions) for each one: three datasets for training and one dataset for testing.

\section{METHOD}

The method used here to improve BCI classification accuracy based on assigning a class to a complete sequence of instances is based on two main ideas. First, the transition in the signal from a class to another class must be detected. This knowledge is used to discard the class assigned to the previous sequence of instances, just before the transition. Hence, the prediction problem of $N$ classes is transformed into a prediction problem of $N-1$ classes. Usually, in classification tasks the reduction of the number of classes helps to increase the performance of the classification algorithm.

On the other hand, the proposed method is inspired in the idea that in this problem, the class to predict remains fixed for a time period that we have called sequence of instances between transitions. That means that, within a sequence, the class is not continuously changing. Hence, it makes sense to try to guess the class assigned to that sequence instead of using the classifier to predict each instance of the sequence independently of each other. The simplest way to assign a class to a sequence is to compute the majority class returned by the classifier on a small set of instances at the beginning of the sequence. However, if the classifier makes many mistakes at the beginning of the sequence, the whole sequence will be missclassified. In our first approach, we tried to assign a class to whole sequences based only on the first instances, and very frequently, complete sequences were missclassified, and the whole method crashed.

Therefore, we have decided to classify the $i^{\prime} t h$ instance in the sequence by considering a moving window of the $n$ previous instances. This gives some inertia to the classifier (it considers $n$ instances instead of just one), but if mistakes are made by the user, the classifier will recover eventually, once the moving window has gone past the mistaken instances. We offer a well-founded method for selecting the window size $n$, that it is explained at the end of the section.

Next, a detailed description of the method is presented. It includes the following parts: the procedure to detect the transition; the procedure to select the classifier once the transition is detected and one of the class is discarded; the procedure to classify test 
instances with a moving window; and, the mechanism to compute the window size.

As we described in Section 2, we assume that the original data in the time domain has been transformed to Power Spectral Density (PSD) in the frequency domain. Thus, we assume that we have a set of samples (or instances) and every sample contains all the components of the PSD for all channels at every instant. That is:

$$
\text { sample }_{i}=\left\{P S D_{r, j}(i) j=1, \ldots, N o C, r=1, \ldots R\right\}
$$

where $P S D_{r, j}(i)$ is the value of the $r^{t h}$ spectral component for the the $j^{\text {th }}$ channel, NoC is the number of channels, and $R$ is the number of components in the PSD. For instance, if the PSD ranges from $8 \mathrm{~Hz}$ to $30 \mathrm{~Hz}$ and there is a component every $2 \mathrm{~Hz}$ (a $2 \mathrm{~Hz}$ resolution), $R=12$.

Next, we describe the components of our method:

- Transition detection. It is based on the observation that when a change of class occurs, a change in the frequency domain also occurs. The idea is to detect that transition by computing the Euclidean distance between PSDs at two consecutive samples. For every sample $i$, the distance $d_{i}=\|P S D(i)-P S D(i-1)\|$ between the PSD of sample $_{i}$ and sample ${ }_{i-1}$ is calculated as:

$$
d_{i}=\sum_{j=1}^{N o c}\left(\sum_{r=1}^{R}\left|P S D_{r, j}(i)-P S D_{r, j}(i-1)\right|\right)
$$

Once the distance $d_{i}$ is obtained, a threshold value $U$ has to be set, such that if the distance is higher that this threshold, a transition is signaled. The value of $U$ is crucial for successfully predict transitions and therefore, very important for the success of the method.

In order to determine that threshold, the following mechanism is applied. The training data set $X$ is divided into two subsets, named $X_{\text {notransition }}$ and $X_{\text {transition. }}$. The first one, $X_{\text {notransition, con- }}$ tains the samples for which there is no transition; and, the second one $X_{\text {transition }}$ contains the transition samples. This separation can be made because samples are labeled in the training set (i.e. their class is known), and therefore a transition is just a change of class. After that, the distances $d_{i}$ for samples in $X_{\text {notransition }}$ are calculated and the maximum of these dis-

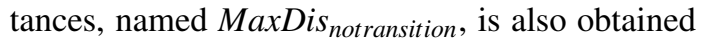
- The distances $d_{i}$ for samples in subset $X_{\text {transition }}$ are also obtained and ranked from low to high. Obviously, a good threshold $U$ must be larger than MaxDis notransition. We currently define $U$ as the next $d_{i}$ of set $X_{\text {transition }}$ that is larger than MaxDis notransition. This way of setting the threshold does not guarantee that all transitions will be detected. However, this method is simple and reasonable and later we will show that it performs correctly on the test data.

- Choosing the appropriate classifier, once the transition has been detected. Let us remember that when a transition is detected, the class of the previous sequence of instances is discarded (i.e. after the transition, the class must change). Therefore, after the transition, the system only needs a classifier that is able to classify samples (or instances) into $N-1$ classes (discarding the previous class). As the system must be prepared for all classes, all $(N-1)$-class classifiers must be trained off-line using the training data, prior to the system going on-line. If there are $N$ classes $\left(C_{1}\right.$ to $\left.C_{N}\right)$, there will be $N(N-1)$-class classifiers. The first classifier, named $K_{2, \ldots, N}$, assumes that class $C_{1}$ is discarded and will predict the class in the set $\left\{C_{2}, \ldots, C_{N}\right\}$. The second classifier, named $K_{1,3, \ldots, N}$, will discard class $C_{2}$ and classify instances in $N-1$ classes in the set $\left\{C_{1}, C_{3}, \ldots, C_{N}\right\}$. The $k^{\prime} t h$ classifier, named $K_{1, \ldots k-1, k-1, \ldots, N}$, will consider only classes in the set $\left\{C_{1}, \ldots, C_{k-1}, C_{k+1}, \ldots, C_{N}\right\}$. And so on, until $\left\{C_{1}, \ldots, C_{N-1}\right\}$. Also, an $N$-class classifier must be learned, because at the beginning there is no previous sequence of samples, and therefore, no class can be discarded. In this work, we have used Support Vector Machines (SVM) because they obtain a very good performance(Vapnik, 1998), although any other machine learning algorithm could have been employed. ${ }^{3}$

The method is applied to predict the class in real time (on-line). The main idea is to use one of the classifiers for $N-1$ classes, when a transition is detected, instead of using the general classifier (the classifier for $N$ classes). Usually, the performance of classifiers with fewer classes is higher because if a class is removed, uncertainty about the class is also reduced.

The procedure to select the classifier in real-time is described next: when the prediction process begins, the N-class classifier is responsible for prediction. At the same time, the distances $d_{i}$ given by equation 2 are calculated. When a transition occurs (i.e. $d_{i}>U$ ), one of the $N$ classifiers for $N-1$ classes must be chosen. To choose the

\footnotetext{
${ }^{3}$ In fact, we use Weka's SMO implementation with standard parameters (a linear kernel and $C=1$ (Garner, 1995).
} 
most appropriate classifier, the majority class predicted in the previous sequence of instances (i.e., the set of instances between the last two transitions) must be discarded. Let's suppose that the class of the last sequence was $k$. Therefore, class $k$ will be ruled out for the current transition, and the $K_{1, \ldots k-1, k-1, \ldots . N}$ classifier will be responsible for prediction during the current sequence until the next transition is detected.

\section{- Procedure to classify test instances with a mov-} ing window

As explained at the beginning of the section, we use a second idea here to improve classification accuracy: in order to classify sample $e_{i}$, a window with the predictions of the selected classifier for the $n$ previous samples will be used, instead of

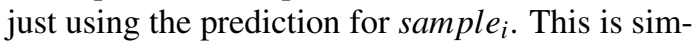
ilar to determining if a biased coin is biased towards heads based only on the $n$ previous few coin tosses. Sample $e_{i}$ will be classified as the majority class of instances within the window. It is a moving window because only the last $n$ predictions just before sample $_{i}$ are taken into account. As the windows moves, all the samples inside it are classified by the selected classifier. Figure 1 shows how it works. The only remaining issue is to estimate a "good" window size $n$. This will be done next.

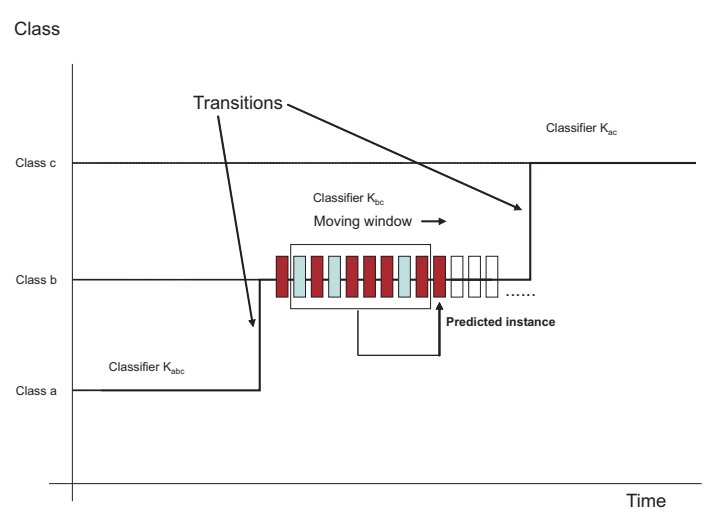

Figure 1: Moving window to classify test instances

\section{- Computing the size of the moving window}

Assigning the majority class of instances within the window to the next sample is reasonable, but mistakes would occur if the frequency of that class in the window is not too far from $50 \%$. This can be solved by establishing a safe confidence interval around the estimated frequency. For simplification purposes, let's suppose there are only three classes $(N=3$, classes $a, b$, and $c$ ), and as explained before, one of them will be discarded after the transition and one of the 32 -class classifiers will be used for the current sequence until the next transition. Let's suppose that class $a$ is discarded and therefore classifier $K_{b c}$ must be used for the current sequence ( $K_{b c}$ separates class $b$ from class $c$ ). Although, the class of the current sequence is fixed until the next transition, the predictions of $K_{b c}$ will make mistakes. In fact, just like coin tosses, classification errors follow a $\mathrm{Bi}$ nomial distribution (with success probability $p$ ). If the actual class of the current sequence is $b$, the distribution of mistakes of $K_{b c}$ will follow a Binomial distribution with $p=T P_{K_{b c}}^{b}$, where $T P_{K_{b c}}^{b}$ is the True Positive rate for class $b$ and classifier $K_{b c}$ (i.e. the accuracy for class $b$ obtained by classifier $K_{b c}$ ). On the other hand, if the actual class is $c$, $p=T P_{K_{b c}}^{c}$.

If the actual class is $b, p$ can be estimated $(\hat{p})$ from a limited set of instances (we call it 'the window'), and from standard statistical theory (and by assuming the Binomial distribution can be approximated by a Gaussian), it is known that $\hat{p}$ belongs to a confidence interval around $p$ with confidence $\alpha$ :

$$
\hat{p} \in p+-z_{\alpha} \sqrt{\frac{p(1-p)}{n}}
$$

where $n$ is the size of the window. From Eq 3, we can estimate the size of the window required:

$$
n \geq z_{\alpha}^{2} \frac{p(1-p)}{(p-0.5)^{2}}
$$

When generating predictions, the actual class of the current sequence is not known, and therefore we have to assume the worst case, that happens when $p$ is closest to 0.5 . Therefore, $p=\min \left(T P_{K_{b c}}^{b}, T P_{K_{b c}}^{c}\right)$. To be in the safe side, for this paper, we have decided to make the window size independent of the classifier assigned to the current sequence. Therefore, if there are three classes $p=$ $\min \left(T P_{K_{b c}}^{b}, T P_{K_{b c}}^{c}, T P_{K_{a b}}^{a}, T P_{K_{a b}}^{b}, T P_{K_{a c}}^{a}, T P_{K_{a c}}^{c}\right)$. A similar analysis could be done for more than three classes.

It is important to remark that Eq 4 is only a heuristic approximation, since instances in a window are not independent in the sense required by a $\mathrm{Bi}$ nomial distribution. For instance, if the user becomes tired and unfocused, s/he will generate a sequence of noisy data that will be labeled uncorrectly. If the window includes that sequence of mistakes, the classification based on that window 
will also be mistaken. However, the classifier will recover once the moving window goes past the sequence of mistakes.

\section{RESULTS}

The aim of this Section is to show the results of our method on the datasets described in Section 2. Let us remember that there were three subjects, and each one generated four sessions worth of data. The first three sessions are available for learning while session four is only for testing. All datasets are three-class classification problems with classes named 2, 3, and 7.

Our method computes all two-class SMO classifiers. SMO is the Weka implementation of a Support Vector Machine. Table 1 displays the results of all two-class classifiers $\left(K_{23}, K_{27}, K_{37}\right)$ and the threeclass classifier $\left(K_{237}\right)$. The training has been made with sessions 1 and 2 instances and the testing with session 3. The three-class classifier accuracies can be used as a baseline to compare further results. In brackets we can observe the True Positive rate (TP) for each class. For instance, 74.7 is the True Positive rate (TP) for class 2 for the $K_{23}$ two-class classifier (i.e. $T P_{K_{23}}^{2}$ ).

Table 1: Accuracy of two-class and three-class SMO classifiers for subjects 1,2 , and 3. Training with sessions 1 and 2, and testing with session 3.

\begin{tabular}{|c|c|c|c|}
\hline $\begin{array}{c}\text { SMO } \\
\text { Classifier }\end{array}$ & Subject 1 & Subject 2 & Subject 3 \\
\hline$K_{23}$ & 79.0 & 71.9 & 52.3 \\
& $(74.7 / 83.8)$ & $(68.2 / 75.3)$ & $(53.3 / 51.4)$ \\
\hline$K_{27}$ & 82.4 & 74.3 & 57.9 \\
& $(64.8 / 93.9)$ & $(62.7 / 81.9)$ & $(50.6 / 65.2)$ \\
\hline$K_{37}$ & 83.0 & 76.8 & 60.4 \\
& $(81.2 / 84.4)$ & $(63.5 / 87.7)$ & $(54.9 / 65.9)$ \\
\hline$K_{237}$ & 73.8 & 62.0 & 40.9 \\
\hline
\end{tabular}

Section 3 gives the details for computing the thresholds for detecting transitions. These are: $U_{1}=$ $0.563108963, U_{2}=0.58576, U_{3}=0.587790606$, for subjects 1,2 , and 3 , respectively.

Our method uses the TP rate (class accuracy), obtained with session 3 , for computing the moving window size, according to $\mathrm{Eq} 4 . \quad p$ will be set as the minimum of all TP rates. So we have $p_{1}=0.648$, $p_{2}=0.627$, and $p_{3}=0.506$ for subjects 1,2 , and 3 , respectively. A confidence interval with $\alpha=0.99$ will be used, therefore $z_{\alpha}=2,5759$. Table 2 displays the windows sizes for every subject. It can be seen that the window size for subject 3 requires 46072 instances many more than available, so we apply the window moving idea only to subjects 1 and 2 . This is due to the accuracy of classifiers for subject 3 are very low, in particular the accuracy of $K_{23}$ (see Table 1 ). We have also computed the window size for larger probabilities to check the performance of the method if smaller window sizes are used. For instance, we have also considered $p_{1}=0.80$ and $p_{2}=0.70$ (those values are approximately the accuracies of the twoclass classifiers in Table 1).

Table 2: Window size used for subjects 1, 2, and 3.

\begin{tabular}{|l|c|c|}
\hline & Probability & Window Size \\
\hline Subject 1 & 0.648 & 78 \\
Subject 2 & 0.627 & 92 \\
Subject 3 & 0.506 & 46072 \\
\hline Subject 1 & 0.80 & 12 \\
Subject 2 & 0.70 & 92 \\
\hline
\end{tabular}

Finally, Table 3 shows the final results. The first row displays the competition results (Galan et al., 2005) on session four. (Galan et al., 2005) proposed an algorithm based on canonical variates transformation and distance based discriminant analysis combined with a mental tasks transitions detector. As required by the competition, the authors compute the class from 1 second segments and therefore no windows of samples are used. The second row, displays the best results from the competition using longer windows (Gan and Tsui, 2005) (it reduces dimensionality of data by means of PCA and the classification algorithm is based on Linear Discrimination Analysis). No details are given for the size of the window of samples. The third row shows the results of the three-class classifier (sessions 1 and 2 were used for training and session 4 , for testing). The fourth row contains the results (on session four) of applying the transition detector only. In this case, once the transition is detected, the previous class is discarded and the prediction is made using the two-class classifier chosen. These results are better than the threeclass classifier. For subject 3, the performance of the method using the transition detection is very low because some of the two-class classifiers for subject 3 have a very low accuracy (see $K_{23}$ in Table 1). Let us remember that when a transition is detected, the previous class is discarded. The previous class is computed as the majority class of the previous sequence. If the classifier used in the previous sequence is very bad, the majority class might not be the actual class. Hence, the wrong class would be discarded in the current sequence, and the wrong 2-class classifier would be selected. That would generate more mistakes that 
would be propagated into the next sequence, and so on. Given that all samples between transitions are used to compute the previous class and that the $(\mathrm{N}$ 1) classifiers are better than chance, the possibility of mistaking the previous class is very low. In fact, for the data used in this article, this situation has not occurred. But it is important to remark that preventing the propagation of the missclassification of the previous class is crucial for the success of the method and we intend to improve this aspect in the future.

The fifth row in Table 3 shows the results with the method described in Section 3. In this case, both ideas, the transition detection and the moving window size, are used. Results improve significantly if the moving window is used: classification accuracy raises from 74.8 to 94.8 and from 74.6 to 86.3 (subjects 1 and 2, respectively). It can also be seen that results are also improved if a smaller window size is used (MW with small sample).

Comparing our method with the best competition result that used a window of samples (second row of Table 3), we can see that our method is competitive with respect to the first subject and improves the performance for the second subject. Unfortunately our method cannot be applied to the third subject due to the large number of samples required for the window. It can also be seen that using windows improves accuracy significatively (second, fifth, and sixth rows versus the first one of Table 3).

Table 3: Results for subjects 1, 2, and 3.

\begin{tabular}{|c|c|c|c|}
\hline & Subj. 1 & Subj. 2 & Subj. 3 \\
\hline BCI comp. (1 sec.) & 79.60 & 70.31 & 56.02 \\
BCI comp. (long window) & 95.98 & 79.49 & 67.43 \\
3-class classifier & 74.8 & 60.7 & 50.2 \\
Transition detector & 80.8 & 74.6 & 52.2 \\
Moving window & 94.8 & 86.3 & - \\
MW small sample & 84.2 & 82.5 & - \\
\hline
\end{tabular}

\section{SUMMARY AND CONCLUSIONS}

Typically, in BCI classification problems EEG samples are classified at every instant in time independently of previous samples. These samples run in sequences belonging to the same class, and then followed by a transition into a different class. We present a method that takes this fact into consideration with the aim of improving the classification accuracy. The general classification problem is divided into two subproblems: detecting class transitions and determining the class between transitions. Class tran- sitions are detected by computing the Euclidean distance between PSD at two consecutive times; if the distance is larger than a certain threshold then a class transition is detected. Threshold values are automatically determined by the method. Once transitions can be detected, the second subproblem -determining the class between transitions- is considered. First, since the class before the transition is known, it can be discarded after the transition and therefore, a Nclass problem becomes a (N-1)-class problem, which is easier. Second, sequences between two transitions may contain many instances but only a few are necessary to determine the class of the whole sequence. In order to determine the class between transitions a moving window is used to predict the class of each testing instance in such a way that only the $n$ last predictions before the testing instance are taken into account. The estimation of the window size $(n)$ is based on standard statistical theory.

This method has been applied to a high quality dataset which had been previously precomputed, resulting in a three-class classification problem with 96 input attributes. These data corresponds to three subjects, with four sessions for each one: three for training and one for testing. Several experiments have been done in order to validate the method and the obtained results show that just by applying the transition detector, the classification rates are better than when a 3-class classifier is used. When the moving window is used, the results are significatively better. Those results are also competitive to those obtained in the BCI competition: similar for subject 1 , better for subject 2 , and worse for subject 3 . We also show that if a smaller window size is used the classification rates are also better than those that use only the transition detector and the three-class classifier.

\section{REFERENCES}

Curran, E. and Stokes, M. (2003). Learning to control brain activity: a review of the production and control of eeg components for driving braincomputer interface (bci) systems. Brain Cognition, 51.

Dornhege, G., Krauledat, M., Muller, K.-R., and Blankertz, B. (2007). Toward Brain-Computer Interfacing, chapter General Signal Processing and MAchine Learning Tools for BCI Analysis, pages 207-234. MIT Press.

G. Pfurtscheller, C. N. and Birbaumer, N. (2005). Motor Cortex in Voluntary Movements, chapter 14, pages 367-401. CRC Press.

Galan, F., Oliva, F., and Guardia, J. (2005). Bci competition iii. data set v: Algorithm description. In Brain Computer Interfaces Competition III. 
Gan, J. Q. and Tsui, L. C. (2005). Bci competition iii. data set v: Algorithm description. In Brain Computer Interfaces Competition III.

Garner, S. (1995). Weka: The waikato environment for knowledge analysis. In S.R. Garner. WEKA: The waikato environment for knowledge analysis. In Proc. of the New Zealand Computer Science Research Students Conference, pages 57-64.

Kubler, A. and Muller, K.-R. (2007). Toward BrainComputer Interfacing, chapter An Introduction to Brain-Computer Interfacing, pages 1-26. MIT Press.

Millán, J. (2004). On the need for on-line learning in brain-computer interfaces. In Proceedings of the International Joint Conference on Neural Networks, Budapest, Hungary. IDIAP-RR 03-30.

Millan J del R, Renkens F, M. J. and Gerstner, W. (2004). Noninvasive brain-actuated control of a mobile robot by human eeg. IEEE Trans Biomed Eng, 51.

Vapnik, V. (1998). Statistical Learning Theory. John Wiley and Sons. 